\title{
Second Language Proficiency and Maze: Marathi-English Bilinguals
}

\author{
Rahul Chakraborty, Nicole Morales, Kendell Fritsch, Maria Diana Gonzales \\ Department of Communication Disorders, Texas State University, Texas, United States
}

This study examined influence of age of academic $L 2$ exposure on production of mazes in two groups of Marathi-English bilinguals varying in their English proficiency. Thirty- two adult bilingual Marathi (L1)-English (L2) speakers described three different culturally-calibrated picture cards in L2. The participants varied in ages of initial academic L2 exposure and proficiency and formed two different L2-proficiency groups. From their descriptions, pauses, repetitions, and revisions were analyzed. Results suggest that the participants with early age of L2 exposure and high L2 proficiency (early/high group) produced less number of mazes and fewer repetitions than the participants with late L2 exposure and low L2 proficiency (late/low group). The number of mazes and the types of mazes varied with the age of L2-exposure. When, findings are compared to our previous work on maze and L2-proficiency relationship, clearly maze production varies with the target language, $\mathrm{L} 2$ proficiency and the target tasks.

Keywords: Marathi, Bilingualism, Maze, Picture description, L2 Proficiency

\section{INTRODUCTION}

Producing two languages often imposes a relatively higher cognitive-linguistic load than producing one language [1-3]. Any increase in processing load might disrupt the normal speech production mechanism, and might be overtly realized as mazes $[4,5]$. Mazes are considered as interruptions in the forward flow of speech and have been described as "...a series of words (or initial parts of words), or unattached fragments which do not constitute a communication unit and are not necessary to the communication unit" [6, p. 22]. It is generally accepted that production of mazes reflect speakers' uncertain responses to the linguistic demands while processing language specific tasks. Mazes are also a consequence of the speaker's simultaneous covert repair operations while monitoring through the perceptual-loop [5]. Mazes of different types are surfaced in speakers' production presumably due to three independent mechanisms: (a) attempt to control the context-ambiguity of the content, (b) attempt to control the syntactic and phonological patterns, and (c) attempt to control the synergy between speakers' intention and production [5,7,8]. Generally, the two highly agreed upon underlying reasons for maze production are, an increase of plan-time to finish an unfinished utterance [9] and to repair an undesired construct [10]. Thus mazes capture restoration activity involving phonology, morphology, syntax, and semantics [e.g., 8,11-13] and the

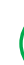
$(G-1)$

Received: June 20, 2017

Revision: September 2, 2017

Accepted: September 4, 2017

Correspondence:

Rahul Chakraborty

Department of Communication Disorders, 601 University Drive, Texas State University, San Marcos, Texas 78666, United States

Tel: (512) 245-6577

Fax: (512) 245-2029

E-mail:rc39@txstate.edu

(C) 2017 The Korean Association of SpeechLanguage Pathologists

This is an Open Access article distributed under the terms of the Creative Commons Attribution Non-Commercial license (http:// creativecommons.org/licenses/by-nc/4.0/) which permits unrestricted non-commercial use, distribution, and reproduction in any medium, provided the original work is properly cited. 
neurophysiological processes [e.g., 5,7,8]. Historically, mazes have been associated with language development and linguistic proficiency [6]. In addition, mazes are also known to vary with speaker, content, language, or even dialect [e.g., 14, $15,17]$. In this paper, we examine influence of L2 proficiency on maze production in bilingual Marathi-English adults.

\section{Maze Production}

Mazes appear at both grammatical intervals (i.e., at punctuations or before relative and interrogative pronouns) and at ungrammatical junctures (i.e., in the middle of a phrase or at the beginning of a sentence) [18]. Grammatical mazes, which constitute $55 \%$ of the total maze frequency, occur naturally and do not interfere with the listener's comprehension. Ungrammatical mazes, constituting the remaining $45 \%$ of the total mazes, usually result in repetition of phrases [18]. Most mazes are produced at pre-syntactic junctions as speakers undergo planning for the incoming phrase or constructs [19]. A maze before a noun potentially suggests that a speaker is adding time to find the correct word or trying to modify the noun, or attempting to correct a potential/anticipatory misarticulation $[9,10]$. However, a maze before a verb is observed when a speaker either looks for the desired verb or tries to modify the verb [10]. Speakers are also known to use mazes when they feel unsure of their intended construction, to ensure appropriation of pragmatic norms as observed in turn taking and also to ensure listeners focus [19].

Usually, two theories are used to explain speakers' mazing behaviors and restoration attempts. Kapatsinski's (2010) Gradient Continuity Hypothesis suggests that speakers usually want to produce uninterrupted utterances and they repair a word-maze only after finishing the word. The Main Interruption Rule [20] claims that speakers repair an error as soon as it is detected and do not always wait for the word to be finished in the production chain. High frequency words are less likely to be revised during production and are usually not revised until after the word is produced [21]. Low frequency words are revised the moment an error is detected and speakers don't usually wait until the end of the production [21].

Speakers are known to produce various types of mazes. Some mazes are time-dependent, as in empty and filled pauses. Empty pauses are silent intervals, two or more seconds in length [22] and filled pauses are non-linguistic vocalization at the beginning of utterances or between words [23]. Some mazes involve recursivity, as observed in sound repetition (i.e., repeating a phoneme), part-word repetition, whole- word repetition and phrase repetitions. Some mazes involve overt correction trials as in phrase revisions, lexical revisions, and grammatical revisions. Some mazes are classified as connectors when repetitive use of conjunctions or time-markers is observed at the beginning of utterances. Production of mazes and their types have been used as a window to explore language learning and language production difficulty [5].

Production of mazes has been associated with both normal and abnormal speakers of different languages [23]. Increased production of mazes has often been associated with impoverished language proficiency and language impairment $[6,16$, $23,24]$. For example, excessive maze production may be an indicator of a language-learning problem [25]. In their study, only content mazes were produced in higher frequency compared to filled pauses and the children with Specific Language Impairment (SLI) used fewer filled pauses than children with normal language (NL) controls. Children with SLI as well as typically developing (TD) children predominantly use pauses and repetitions throughout the narrative sample [8]. Miller (1996) had reported that a higher percentage of mazes in school-aged children frequently suggest a potential language disorder. Children with SLI are known to produce significantly more mazes compared to their mean length utterance matched control group [22].

Even though maze production could be used as an index of language processing mechanisms and children with language disorders are known to use higher number of mazes compared to their age matched normal peers, some researchers have warned against singularly/linearly associating higher frequency of mazes to a possible language disorder $[4,22,23$, 26]. It is common for typically developing (TD) children to use more pauses during their narrative retells [8]. Even normal adult bilinguals use higher number of mazes compared to monolinguals and bilinguals maze more in their non-dominant language [27]. Mazes have a diagnostic relevancy pertaining to language impairments in children, however it is unclear how that translates to adult bilingual speakers.

All speakers produce mazes, bilinguals more than the monolinguals [28]. Since bilinguals choose their required constructs from two functional language systems to a variable degree, words or other required linguistic constructs/components are activated in both languages. The Interference Hypothesis [28] attempts to explain why bilinguals tend to maze more than their monolingual peers. According to the Interference Hypothesis, since bilinguals have two different sets of language-specific rules, the target constituents are activated 
in both languages and the resultant competition between the two systems give rise to more mazes compared to monolingual speakers who only need to select the target constituents from one linguistic system [28]. Compared to the monolinguals, bilinguals are known to produce more mazes in their non-dominant or relatively less proficient language (which might not be their L2) [29], and more in L2 than in their L1 [11, 13,30-34].

Usually, maze production has been reported to vary over time with the target language [35]. For example, comparison of maze use between English and Spanish narratives in school-age bilingual children learning English had indicated that maze use significantly varied over time between English and Spanish with a relatively plateaued trajectory observed in maze use in English but a downward trajectory in Spanish maze scores [35]. Similar results were reported earlier with higher number of mazes observed in L2 than in L1 [11,13,33, $34]$ and stable trajectory of maze use over time in monolingual English speaking children $[6,24,36]$. As a potential explanation for the difference observed in maze production between English and Spanish, as L2 and L1 respectively, and the decreasing trend of maze scores in Spanish, it has been hypothesized that speakers, over time, required less repair strategies (mazes) as English proficiency increases [1]. Simultaneously, increased language proficiency and familiarity with the L1, Spanish, speakers might reduce their cognitive-processing load, which results in a decrease in maze production [1].

Frequency of mazes is known to be proportional to the general complexity of language being used with an increase in the maze-frequency proportional to the complexity of the target language [24]. A higher maze frequency in complex sentences pertains to syntactic complexity rather than to narrative complexity [35]. Leadholm and Miller (1992) had reported that participant-specific variability in maze production was observed when children attempted to express complex ideas. Even Hopper (2014) had reported that the use of reduced language-interference could be an explanation to account for a greater difference in maze production between simple and complex sentences in English than in Spanish. It is historically presumed that language interference tends to occur more with non- dominant language production than with dominant language production [29]. This interference theory explains why consistently researchers' reported more mazes in the nondominant language $[6,24,35,36]$.

Maze frequency and types might vary with the target language as mazes are influenced by the semantic load, the length of words, and the level of syntactic complexity [1]. As reported earlier for bilingual speakers with variable language proficiency in L1 and in L2, language specific phonological, morphological, semantic, syntactic and pragmatic properties might differentially influence speakers $[4,29]$.

Mazes are usually more frequent during narrative retells and stories in comparison to conversation [8]. In both L1 and L2, but more in L2, relatively higher frequency of mazes are observed in complex sentences than in simple sentences and hence it is accepted that the nature of utterance complexity and L1 vs. L2 can also influence maze production [35,37]. For example, in a narrative story, speakers allocate a significant amount of attentional resources to conceptualize the narrative [5]. The thematic content, the complexity of the picture, speakers' proficiency in the language of narration and the nature of the task are likely to impose a processing load on speakers' overt production mechanism. Hence, in a bilingual population, mazes as an index of increased processing load are frequently observed in all speakers to a variable degree $[38,39]$.

However, mazes are not always generated to mark a lack of linguistic proficiency as a potential surface representation of the underlying processing load. For example, media professionals deliberately infuse hesitations, as they prefer to speak spontaneously than reading from a list [17]. Mazes even help us understand the underlying production processes [40,41] as we know that words following mazes have low transitional probability, and thus have high information value [18]. This is not exclusive to media professionals and the public speakers, even normal speakers infuse mazes at times to attract their audience. For example, even though pauses are considered a type of maze, it can pre-signal to an upcoming important linguistic content [e.g., 42,43]. Hence, mazes should not be indiscriminately associated with language impairment or a lack of language proficiency by drawing parallel between 'spontaneous' speech and 'functionally inadequate' speech [44]. Mazes, in spontaneous speech, can even be unnoticed (e.g., 45].

Even though bilingual speakers are known to produce more mazes in their nondominant language, early ages of exposure to an L2 usually offers advantages to bilinguals in various linguistic tasks [46-50]. It is widely accepted that an early age of exposure to an L2 offers an advantage to bilingual speakers on L2 production [48,51-53]. An early age of exposure to an L2 offers advantages to bilinguals even in minimizing production of mazes.

However, while some studies have endorsed a bilingual disadvantage by analyzing speech disfluencies [54-57], other 
studies have indicated minimal to no differences between bilingual and monolingual in verbal fluency [23]. For example, Bedore et al. (2006) reported that maze types were similar between Spanish-English bilingual children speaking in English or in Spanish. There have been additional reports where after a year of school, no monolingual advantage was observed when bilinguals were compared [58-60]. Adult bilinguals offer an interesting test scenario since producing two languages might impose a higher cognitive/processing load than producing only one language [1].

Adults with early L2 exposure certainly have an advantage in their linguistic experience compared to speakers with late L2 exposure. In general, the frequency of L2 input, speech motor practice, cognitive-linguistic processes in L2 and extended social usage of L2 help differentiate the two groups of bilinguals. Thus potential differences in mazing behaviors between the high and the low L2-proficiency groups could stem from variations in speakers' linguistic experience and variations in language processing [4]. The current paper explores the relationship between age of initial L2 exposure and L2 proficiency on mazing behaviors through spoken narratives of bilingual adults in their non-dominant language, L2.

However, even though mazes are considered a potential index of linguistic processing skills, maze production in bilingual adults have not received sufficient empirical attention [23]. To explore mazing behaviors in adult bilinguals, Chakraborty et al., (2011) examined influence of age of academic L2 exposure and L2 proficiency on maze production. Results suggested that age of academic L2 exposure and proficiency did not influence the overall frequency of mazes, in the two groups of bilinguals who differed in their age of academic L2 exposure and L2 proficiency. However, among all the maze-types, the two proficiency groups differed only, in their use of empty pauses; the early L2 exposed and high L2 proficiency group used a relatively smaller number of empty pauses compared to those bilinguals who were academically exposed to an L2 late and had a relatively low $\mathrm{L} 2$ proficiency.

\section{The current study}

The current study is an extension of our previous work where we had examined the influence of age of academic L2 exposure and L2 proficiency on maze production in 2 groups of Bengali-English bilinguals varying in their age of academic L2 exposure and proficiency [4]. The results of our previous study indicated that the two proficiency groups differed only in their use of empty pauses; the early L2 exposed and high L2 profi- ciency group used a relatively smaller number of empty pauses compared to those bilinguals who were academically exposed to an L2 late and had a relatively low L2 proficiency. Using the same methodology and including participants from the same country, we wanted to explore if participants with a different L1 background but with similar difference in age of academic L2 exposure and L2 proficiency, exhibit similar maze behaviors while performing the same narrative tasks that were included in Chakraborty et al., (2011). The authors suggested against linearly overgeneralizing maze and L2 proficiency relationship, as the types of mazing behavior appeared far more complex and reticulated than what they had originally hypothesized [4].

The current study compared maze use in two groups of adult bilinguals who use Marathi as their first language (L1) and English as their L2, but only differed in their initial age of academic English exposure (early vs. late) and oral English proficiency. Marathi-English bilinguals offer a test case because some previous studies have reported that specific maze behaviors are sensitive to the grammatical differences between the two languages being spoken [e.g., 33]. Bengali and Marathi, differ syntactically.

Marathi belongs to the Indo-Aryan branch of the Indo-European family of languages. It is one of the 22 official languages of India and is a state language of Maharashtra, the commercial and entertainment hub of India. In India, there are 73 million who use Marathi as their first language and 3 million people as a second language. According to the US census of 2011, there were 815,345 speakers of Other Indic languages, where Marathi constituted a major portion (specific number is not mentioned in the US Census). Marathi is the primary medium of everyday communication in Maharashtra and is also extensively used in education, government, business, and the media. The Marathi-English bilingual adults offer an interesting extended test scenario to examine the nature of maze behaviors L2 English speakers from a post-colonial environment where English is not the lingua franca, but a major index of socio-academic and economic growth.

We examined the following three questions to explore if and how age of academic L2 exposure and proficiency and mazes interact in adult bilingual speakers: 1) Does frequency of maze use vary as a function of age of academic L2 exposure and proficiency? 2) Do patterns of specific maze type (e.g., pauses, receptions, revisions etc.) vary as a function of age of academic L2 exposure and proficiency? 3) Do patterns of maze production vary as a function of stimuli type? 


\section{METHODS}

\section{Participants}

Thirty-two bilingual adults participated (see Table 1; ages ranged from 24 years to 49 years; $M=27.71 ; 14$ females, 18 males) with Marathi as their L1 and English as their L2. Of the thirty-two participants, seventeen had a history of early academic exposure to English (beginning at elementary school age) and raw scores $18-25, \mathrm{M}=21.6, \mathrm{SD}=3.75$ on the Test of Adolescent and Adult Language-Third Edition (TOAL-3) [61] that indicated high proficiency. The remaining fifteen participants had late academic exposure to English (after 12th grade) and low English proficiency, as indicated by raw scores on the TOAL-3 (3-8, M=7, SD=2.73). Based on the TOAL-3 scores, it is clear that all participants with an early age of academic L2 exposure had high L2 proficiency scores (hence, this group would be labelled as the early/high group) and the fifteen bilinguals who had a late academic L2 exposure had low L2 proficiency scores (henceforth, this group would be labelled as the late/low group). The L2 proficiency scores were obtained to ensure that the two groups clearly differed in their proficiency scores and there was no overlap. All participants reported history of normal speech, language, and neurological development, and passed a pure tone hearing screening at $20 \mathrm{~dB}$ at $0.5 \mathrm{kHz}, 1 \mathrm{kHz}, 2 \mathrm{kHz}, 4 \mathrm{kHz}$, and $6 \mathrm{kHz}$.

All participants were born and stayed in Mumbai, India, until the data collection. Marathi is the lingua franca in Mumbai with Hindi and English being two other dominant language of the state. The two groups were comparable in their academic qualifications as all were college graduates and in their L1 experience. Both the parents of all the participants were native speakers of Marathi. All participants were exposed to English as their L2 at the same age in their school at the elementary school level. However, the two groups had different quality of English exposure in school. The early/high

Table 1. Participants Information

\begin{tabular}{lcc}
\hline Groups & Early/High & Late/Low \\
\hline $\begin{array}{l}\text { Academic qualifications } \\
\text { L1 Experience }\end{array}$ & Master degree & Master degree \\
& $\begin{array}{c}\text { Marathi } \\
\text { since birth }\end{array}$ & $\begin{array}{c}\text { Marathi } \\
\text { since birth }\end{array}$ \\
Parents & Native marathi & Native marathi \\
& speakers & speakers \\
Age range & 24 to 46 years & 23 to 49 years \\
Language of Instruction at \\
school & English & Marathi \\
& since 3 years & after grade 12 \\
\hline
\end{tabular}

group had simultaneous exposure to Marathi and English from the elementary school level, as the medium of instruction in school for the early/high group was English. For the late/low group Marathi was the medium of instruction in school and English language was only a course/subject that focused on grammar and prose. For the late/low group, the language/medium of instruction in academia became English only after grade twelve. All participants reported that they are most proficient in Marathi. The research reported in this manuscript adheres to basic ethical considerations regarding the protection of human participants in research and has been approved by Texas State University's Committee on the Use of Human Research Subjects. Written consent was obtained where participants were told that their identity would remain anonymous and that they could withdraw at any time during the experiment. We choose not to include the monolingual English-speaking control group as there were inherent differences in language processing mechanisms between monolinguals and bilinguals and potential confounds with socioeconomic status (SES), education, and life-experience.

\section{Stimuli and procedure}

The participants were asked to describe one picture and two picture cards in English, participants L2. The order of presentation was fixed to ensure the gradation of nativity and only one picture was presented at a time and no time limit was given. The first picture was the "Cookie Theft" picture taken from the Boston Diagnostic Aphasia Examination [62]. The picture depicted a scene from Western civilization with people, their attires, the setting, and the actions in the picture. The second and the third stimuli were picture sequences based on Aesop's fable [63] and are common to both English and Bengali linguistic heritage. The second picture is based on the story of a lion and a mouse (The Lion), where a lion, who, after catching a mouse, decides to release him. The mouse reciprocates in gratitude, with a comparable life-saving gesture towards the lion. In the third picture (The Fox) a crow loses a piece of food when a fox tempts the crow to open its mouth and sing. All pictures were line drawings and were in black and white. The first picture was completely unknown and nonnative to the participants. The second stimulus was common to both Marathi and English culture, but the attires worn by the hunters in that picture were western. The third stimuli had only animals and hence were culturally neutral. The participants were asked to describe the pictures by telling a story (i.e., "I am going to ask you to describe three pictures. Look at 
each picture. Spend as much time as you want. Describe what you see in the picture."). The audio samples were recorded using PRAAT acoustic software [64].

\section{Measures}

Two graduate students transcribed 96 transcripts (32 participants $\times 3$ pictures/sequences each) from digital audio samples. The graduate students were trained in narrow transcription, and had successfully completed coursework in phonetic transcription and language sample analysis. These two student experimenters used PRAAT acoustic software [64] to analyze productions. For each participant and for each picture sample, the experimenters coded the total number of mazes and different maze types.

The types of mazes coded were: empty pause, filled pause (e.g., Um* she's washing dishes), sound repetition (e.g., The [w*] window is open), part-word repetition (e.g., The [ $\left.\mathrm{pl}^{*}\right]$ plates are in the sink), whole-word repetition (e.g., The [plates*] plates are in the sink); phrase repetition (e.g., [The plates are*] the plates are in the sink), phrase revisions (e.g., The [blates*] plates are in the sink), lexical revisions (e.g., The [sister*] mother is washing the dishes, mother $\rightarrow$ sister), and grammatical revisions (e.g., [She*] The mother is washing the dishes) and connectors (see Table 2). Samples that were transcribed were of variable duration and of variable word/morpheme length. Hence, the total number of mazes and the frequency of each maze-type were converted into a percentage score. To compute the percentage scores, the word count excluded the filled pauses. These computed percentages were examined for inter-rater reliability and for statistical analyses.

\section{Statistical analyses}

The two groups of bilinguals were compared based on the to-

Table 2. Examples of the Types of Mazes

\begin{tabular}{ll}
\hline Maze type & \multicolumn{1}{c}{ Example } \\
\hline Empty pause & 2+ seconds of silence \\
Filled pause & $($ Um) she's washing dishes. \\
Sound repetition & The (w) window is open. \\
\hline Part-word repetition & The (pl) plates are in the sink. \\
\hline $\begin{array}{l}\text { Whole-word repetition } \\
\text { Phrase repetition }\end{array}$ & The (plates) plates are in the sink. \\
\hline $\begin{array}{l}\text { Phrase revision } \\
\text { Lexical revision }\end{array}$ & The plates are) the plates are in the sink. \\
Grammatical revision & The (sister) mother are in the sink. \\
\hline
\end{tabular}

tal number of mazes used, the percentage of pause duration, and the percentage of repetitions and revisions. Several repeated measures ANOVAs were performed. The betweengroup variables were bilingual group status (early L2 exposed with high L2 proficiency vs. late L2 exposed with low L2 proficiency). The within-group variables were types and percentages of mazes. The statistical significance level was set at 0.05 .

\section{RESULTS}

Analyses of the data revealed that the two bilingual groups did differ in their total number of mazes, $F(1,30)=43.1, p=0.00$, $h_{p}{ }^{2}=0.59$. The early/high group produced lesser number of mazes than the late/low group (see Figure 1). A main effect of picture stimuli was observed, $F(2,60)=10.16, p=0.00, h_{p}{ }^{2}=$ 0.25; participants produced significantly lesser number of mazes for the Cookie Theft picture compared to The Fox and The Lion picture sequences. A picture by group interaction was observed, $F(2,60)=8.15, p=0.00, h_{p}{ }^{2}=0.21$. Post-hoc testing (Tukey HSD) revealed that for the Cookie Theft picture the early/high and the late/low groups produced comparable number of mazes. For the two picture sequences, the early/ high group produced fewer mazes than the late/low group, potentially suggesting that the picture sequences are probably more sensitive to capture proficiency specific maze production differences between the two bilingual groups.

Since different participants produced narratives of different length/duration and number of words, we converted the number of maze used by the participants into two different percentage scores; one score was with reference to the total

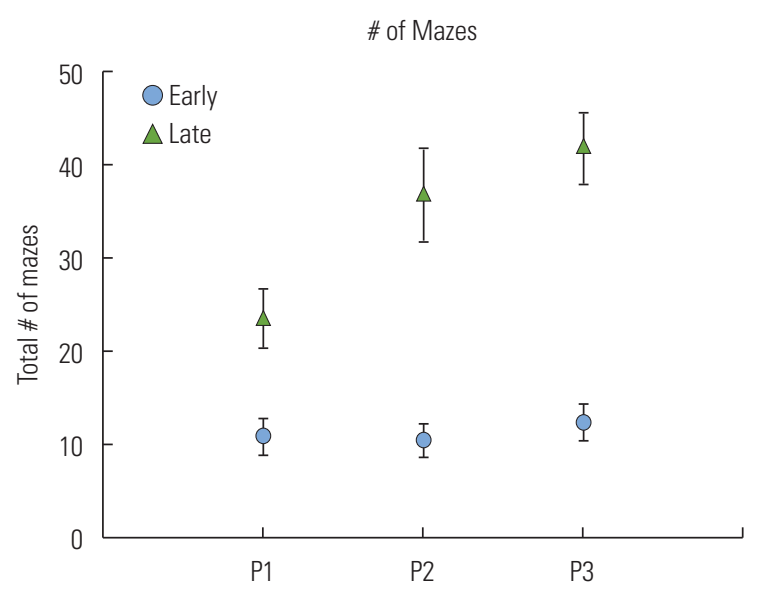

Figure 1. Total number of mazes used by the early/high (filled circle) and late/low (filled triangle) for three pictures; P1 - cookie theft, P2 - the Lion and the Rat; P3 - the Fox and the Crow. Error bars represent standard errors. 


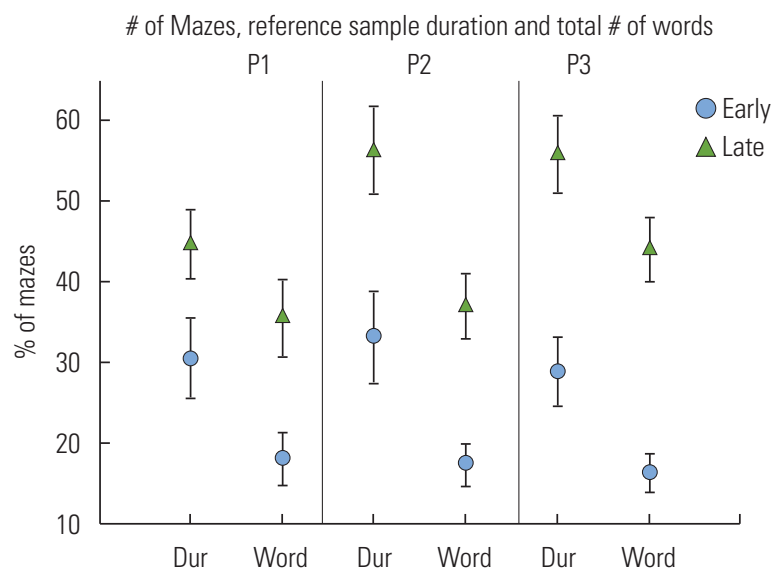

Figure 2. Percentage of mazes used by the early/high (filled circle) and late/ low (filled triangle) for three pictures; left panel P1 - cookie theft, middle panel P2 - the Lion and the Rat; right panel P3 - the Fox and the Crow. Durpercentage based on total duration of the sample, Word - percentage based on the number of words in the sample. Error bars represent standard errors.

duration and the other percentage score was with reference to the number of words. The early/high group used less $\%$ of mazes in relation to the duration of sample than the late/low group, $F(1,30)=11.67, p=0.00, h_{p}{ }^{2}=0.28$ (see Figure 2). The early group used less percentage of mazes, in relation to total number of words within the sample narratives, than the late/ low group, $F(1,30)=29.13, p=0.00, h_{p}{ }^{2}=0.49$ (see Figure 2).

The two groups did not differ in their use of time-dependent mazes (i.e., empty and filled pauses), $F(1,30)=3.25$, $p=0.08, h_{p}{ }^{2}=0.09$. No group by pause-type interaction was observed either, $F(1,30)=0.90, p=0.35, h_{p}{ }^{2}=0.03$. When repetitions were analyzed, the early/high and the late/low groups did differ in their percentage of repetition use, $F(1,30)=16.14$, $p=0.00, h_{p}{ }^{2}=0.35$ (see Figure 3 ). The early/high group used lesser percentages of repetitions compared to the late/low group. There was a repetition type and group interaction, $F(3$, 90) $=5.91, p=0.00, h_{p}{ }^{2}=0.16$. Post-hoc testing (Tukey HSD) revealed that the early/high group did not vary in their production of different repetition types. The late/low group, produced lesser percentage of SR and WWR compared to the percentage of WWR and PR. Similarly, the two groups were not different in their percentage of revisions and connectors, $F(1,30)=0.00, p=0.99, h_{p}{ }^{2}=0.00$. In summary, the two proficiency groups differed in their use of total number of mazes and they also differed in their use of repetitions. The early/ high group used less number of total mazes and lesser percentage of repetitions. In addition, the picture sequences were more sensitive to capture the proficiency difference between the two groups.

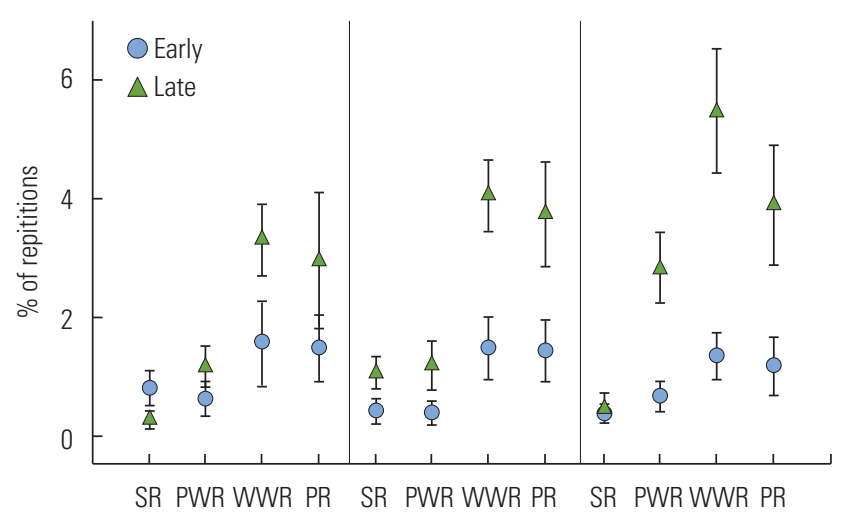

Figure 3. Percentage of repetition used by the early/high (filled circle) and late/low (filled triangle) for three pictures; left panel P1 - cookie theft, middle panel P2 - the Lion and the Rat; right panel P3 - the Fox and the Crow. SR, sound repetition; PWR, part word repetition; WWR, whole word repetition; PR, phrase repetition. Error bars represent standard errors.

\section{DISCUSSION}

The objective of this project was to examine influence of age of academic L2 exposure and proficiency on maze productions of bilingual adults from a post-colonial country. As an initial question, we asked if the overall frequency of mazes was influenced by age of L2 exposure and proficiency. Our results suggested that age of academic L2 exposure and proficiency did induce a discernable influence on the overall frequency of maze productions in the two groups of bilinguals whose L1 was Marathi and L2 was English. Bilingual speakers with early academic exposure to L2 and higher proficiency in L2 (early/high group) produced a lower number of mazes across the stimuli than the participants who were academically exposed to L2 late and had lower L2 proficiency (late/ low group). Specifically, the early/high bilingual speakers produced lesser number of repetitions than the late/low bilingual participants. The two proficiency groups did not differ when revisions, pauses and connectors were compared across the two groups. Finally/Interestingly, among the three stimuli, compared to the widely used cookie theft picture, the two experimental groups differed only in their description of the two picture sequences (i.e., The Lion and The Fox).

There is a prevalent belief that speakers who are more proficient in a language, are more fluent in that language; and hence produce less hesitation. Such hypothesis is extensively supported through various empirical reports with both normal and disordered population (e.g., [65-67]). Some research reports have suggested that proficient speakers of a target lan- 
guage use fewer mazes in that language $[8,23,30,68]$. The current study endorses such findings. The two proficiency groups were different in their mazing behaviors, with the early/high group producing fewer number of mazes compared to the late/low group. The early/high group utilized fewer repetitions across the stimuli than the participants from the late/ low group. The two experimental groups did not differ in their number of revisions used or in their number of connectors used. The early/high group happened to have more neuromotor practice and situational exposure to use L2 compared to the late/low group; hence we observed that the early/high group exhibited fewer production interruptions in the form of mazes.

In addition, the fact that the early/high group used fewer repetitions, and no other form of mazes (revisions, pauses or connectors) captured the proficiency differences between the two experimental groups, suggests that these speakers attempted to repair an error even before the word is completed; the late/low group did such repairs more frequently than the early/high group. Marathi-English bilingual speakers L2 narrative production behavior thus endorses Levelt's (1983) Main Interruption Rule [20] which claims that speakers repair an error as soon as it is detected and do not always wait for the word to be finished in the production chain. The results apparently did not endorse Kapatsinski's (2010) Gradient Continuity Hypothesis because the two groups, who clearly differed in their initial age of academic exposure to English and in production proficiency in English, did not differ in their use of pauses, revisions and connectors. Kapatsinski's (2010) Gradient Continuity Hypothesis suggests that speakers usually want to produce uninterrupted utterances and they repair a word-maze only after finishing the word. In the current study speakers' proficiency-sensitive production behaviors did not wait until after the word was finished; hence repetitions exhibited the proficiency difference. Any proficiency specific difference in the two experimental groups was not even observed in pauses or connectors.

Historically, the production of mazes has been used as a window to understand speakers' planning mechanism and is also considered as overt marker of a potential overload of the production system [18]. The results of the current study suggested that the different types of mazes could be differentially influenced by age of academic L2 exposure or proficiency. The current results also revealed that both the early and the late exposure groups utilized more lexical revisions (e.g., correcting word choice errors) and more connectors than either phonological or grammatical revisions. The underlying mechanisms are potentially different for all these mazing mechanisms.

However, while some previous research reports and the current study have suggested that proficient speakers use fewer mazes than the less proficient speaker of a target language $[8,23,30,68]$, others have not noted significant differences between proficient and less-proficient speakers $[15,23$, 58-60]. Even mature speakers sometimes produce excessive mazes in their L1, despite having advanced language skills [15].

Clearly, the relationship between age of L2-exposure-proficiency and maze-use is highly variable and definitely depends on the L1 of the participants. We suggest such variability in adult linguistic behaviors is because of the following three reasons. First, in our previous study with adult bilinguals from Bengali L1 background, using the same methodology, the early/high group and the late/low group did not differ in their overall production of mazes [4]. In both the studies bilingual participants were born and brought up in the same country, India, and their age of initial exposure and the nature of exposure to L2, English, were comparable across the two studies. But in the current study, the early/high group produced fewer mazes compared to the late/low group. Second, in our previous study with Bengali-English adult bilinguals, based on the same methodology, the two L2 proficiency groups only differed in their use of empty pauses, but in the current study the participants only differed in their use of repetitions. Third, in our previous study, participants did not exhibit different mazing behaviors across the three pictures [4], but in the current study, description of picture sequences appeared to be the more sensitive tasks to capture differences in maze-use patterns between adult Marathi-English bilingual speakers with varying ages of initial L2-exposure and proficiency. However, overgeneralization is cautioned; similar to our previous work [4], we reiterate that the interpretation of the results should be considered with this specific linguistic population, the tasks, and to the method of analyses.

Even historically, language specific variations in mazing behavior is not rare with nested complexities associated with the target language, proficiency of the speakers in the target language and speakers' age. Following four German L1 students studying in an English L2 environment for 24 weeks, Lennon (1990) reported that three out of four students produced more grammatical revisions by the end of the study whereas the frequency of filled pauses and repetitions decreased. Dutch Eng- 
lish L2 students with more experience in L2, English produced more tongue slips [13]. Maze pattern exhibited variability as predominance of mazes shifted from phonological revisions to morphosyntactic revisions as Turkish children's Dutch L2 proficiency increased [69]. Fathman (1980) reported that Korean and Spanish students speaking English as an L2 exhibited varying degrees of self-correction in phonological, grammatical and semantic domains with lexical revisions being the dominant form of maze (40\%). Spanish-speaking students used a lesser frequency of repetitions and revisions compared to the Korean students [70]. It could be hypothesized that selfmonitoring, language proficiency, cross linguistic differences and differences in culture-based concern for conveying correct information contribute to this production variation across languages. Hence, overgeneralizing across languages based on maze production pattern is certainly not recommended in both clinical and non-clinical domains.

Even though narrative details of adult L2 users from multilingual post-colonial linguistic environment has rarely been explored [4], in general, mazes are more frequent during narrative stories and retells [8]. For example, in narrative samples, repetitions and pauses were the most frequently used mazes in children with Specific Language Impairment (SLI) and in TD children [8]. Higher frequency of pauses has been observed in typically developing (TD) children's use of narrative retells [8] and also in adults from multilingual post-colonial linguistic environment [4].

Probing the nature of narrative details further, in the current study, among the three stimuli used to elicit narrative details, the two experimental groups differed in their description of the two picture sequences (i.e., The Lion and The Fox) but not in their description of cookie theft picture. As a task, picture sequences demand a definite beginning, maintenance and conclusion following a specific timeline. Usually, narrators weave a story after evaluating, the number of sequences, the content of each sequence, inter and intra-sequence relationships, and then identify the connecting thread across all those sequences to describe the events chronologically. A picture sequence usually demands a coherent story. However a picture card, without multiple sequences of events, can elicit responses where speakers can only highlight what they can see on the card (e.g., I see a kitchen; I see two kids; The mother is washing dishes). With a picture card the narrator is not required to follow a specific time-line, description does not have to have a beginning-middle-end format, and they do not usually need to present their narration chronologically to create a meaningful sequence of events.

Culturally, the Cookie Theft picture card was reflecting a western style kitchen and both the experimental groups were unfamiliar with that picture as the scene reflected a typical kitchen scene from a western culture. Conversely, the two picture sequences that were used were familiar to the participants as these two stories were common across Indian and American literary culture through Aesop's fables. Hence, it seems, a stimuli equally novel to both the experimental groups might not be sensitive enough to capture the degree of differences in mazing behaviors and age of academic L2 exposure and L2 proficiency. However, it is acknowledged that there is probably a confound as based on this initial attempt it is difficult to conclude if the cultural familiarity aspect captured the maze-proficiency differences across the two groups or the picture-card versus picture-sequences captured the differences. Future studies need to selectively control these culture/familiarity factor and picture card versus picture sequence issues.

\section{Future studies}

In future studies, it would also be interesting to examine using event related potentials (ERP) how the underlying nature of neurolinguistic processes of different maze type vary with reference to specific location within an utterance. Using fMRI and ERP, we could also attempt to explore language-specific maze use to understand neural correlates of different maze types and explore how much of their mazing behaviors are due to bilingual status and how much is due to the characteristics of the spoken languages [23].

In addition, detail analyses of narratives to understand their association with maze production can further our understanding of the inter-relation among linguistic proficiency, speech physiology, and cognitive-linguistic processing. Widely, use Spoken narratives have been considered a more naturalistic and relatively culture-neutral assessment than standardized tasks for culturally and linguistically diverse population [71-73]. However, while doing cross-linguistic experiments, narrative tasks must consider the language variation through measures of dialect density [74-79]. Fine-grained analyses of both macrostructure and microstructure could capture minute details of speakers' linguistic skills as they might relate to language proficiency and maze production. For example, usually in macrostructure analyses speakers are known to consider the beginning (e.g., the person and place are introduced here), the middle of the story (e.g., characters 
encounter conflicts), and the end of the story (e.g., resolution to the problems) $[72,80]$. The narrative macrostructure reflects story grammar [81-86], episodic structure [87,88], high point analysis [89-91], and expressive elaboration [76,92-94]. Simultaneously, speakers' narration could reflect microstructural elements in the way words and sentences are threaded to form a coherent story [80], the semantic and syntactic productivity (e.g., total number of words, total number of utterances), complexity (e.g., number of different words, mean length of utterance), and accuracy (e.g., percentage grammatical utterances). It would be interesting to explore the relative sensitivity of microstructure versus macrostructure to capture mazing behaviors, against the backdrop of the belief that microstructures are more sensitive to developmental differences and are potentially more dialect neutral than macrostructure [95].

\section{Limitations and conclusion}

Clearly, more participants are needed to understand the intricacies and the power of the observed production patterns in this specific linguistic group. This is especially critical because of this specific nonnative population and their relative minority in the USA. A follow up study in the USA is difficult to conduct, as the sociolinguistic environment of the participants would obviously be different in the USA compared to India. Hence, future studies highlighting similar questions need to include more participants and wider variation in the stimulus materials. Clearly, the maze-proficiency relationship needs further exploration using other linguistic groups, as variable findings, even after using the same methodology, across studies could be observed. To minimize the possibility of Type I and Type II errors, future studies should include more linguistic groups with varying L1 backgrounds but with English as their L2. We should include task variations by adding expository samples, delayed imitation tasks and more complex linguistic processing tasks to understand the maze-proficiency relationship. In addition, if we choose to include two monolingual groups, one for English and the other monolingual group from the L1 background of the participants, we might not only get a different reference platforms, but also could compare if maze behaviors in bilinguals are subjected to linguistic transfer. With the increasing number of world Englishes, it is critical to understand how different dialects of English interact with the local L1 in bilingual speakers.

However, in summary, the findings clearly suggest that the age of initial academic L2 exposure and L2 proficiency influ- enced the frequency and types of mazes produced when bilingual Marathi-English speakers described a picture card and two picture sequences in their L2, English. As expected, the early/high group produced fewer mazes overall and fewer repetitions compared to the late/low group. Interestingly, these two groups were comparable across all other maze types.

\section{ACKNOWLEDGMENTS}

Thanks to Amanda Wimsatt for her help with transcription and coding. We also thank Dr. Eugene J. Bourgeois II, Provost, and Dr. Ruth Welborn, Dean of the College of Health Professions at Texas State University, for the research support.

\section{CONFLICTING OF INTERESTS}

The Author(s) declare(s) that there is no conflict of interest.

\section{REFERENCES}

1. Edmunds, P. Buen- -- Buena Gente: Repair in the Spanish of the southwest. Selected proceedings of The 8th Hispanic Linguistics Symposium, ed. Timothy L. Face and Carol A. Klee, 204-213. Somerville, MA: Cascadilla Proceedings Project. 2006.

2. Gutiérrez-Clellen VF, Kreiter J. Understanding child bilingual acquisition using parent and teacher reports. Applied Psycholinguistics. 2003;24:267-288.

3. Silva-Corvalán C. Language Contact and Change. Oxford: Oxford University Press. 1994.

4. Chakraborty R, Domsch C, Gonzales D. Influence of L2 Proficiency on Maze Use. Poster presented at the annual convention of the American Speech, Language and Hearing Association, San Diego, California. 2011.

5. Levelt W. Speaking; From intention to articulation. Cambridge, MA; Bradford Books. 1989.

6. Loban W. Language Development : kindergarten through grade twelve. Urbana, IL: National Council of Teachers of English. 1976.

7. Fletcher P. Evidence from syntax for language impairment. In J. Miller (ed). Research on child language disorders: a decade of progress. San Diego: College Hill Press. 1990.

8. Navarro-Ruiz MI, Rallo-Fabra L. Characteristics of mazes produced by SLI children. Clinical Linguistics and Phonetics. 2001;15 (1 \&2):63-66.

9. Fehringer C, Fry C. Hesitation phenomena in the language production of bilingual speakers: The role of working memory. Folia Linguistica. 2007;41:37-72.

10. Fagan WT. The relationship of the "maze" to language planning and production. Research in the Teaching of English. 1982;85-95.

11. Lennon P. Investigating fluency in EFL; A quantitative approach. 
Language Learning. 1990;40(3):387-417.

12. Postma A, Kolk H, Povel D. Speech planning and execution in stutterers. Journal of Fluency Disorders. 1990;15:49-59.

13. Poulisse N. Slips of the tongue: Speech errors in first and second language production. Amsterdam: Benjamins. 1999.

14. Cruttenden. Intonation Cambridge, Cambridge University Press. 1986.

15. Nippold M. Later language development: School-age children, adolescents, and young adults-3rd Edition. Austin, Texas: ProEd Inc. 2007.

16. Nippold MA. Developmental markers in adolescent language syntax, semantics, and pragmatics. Language, Speech, and Hearing Services in Schools. 1993;24(1):21-28. doi: 10.1044/S016114612401.21.

17. Swerts M. Filled pauses as markers of discourse structure. Journal of Pragmatics 30. 1998;485-496.

18. Goldman-Eisler F. Psycholinguistics: Experiments in spontaneous speech. Boston, MA: Academic Press. 1968.

19. Bortfeld H, Leon SD, Bloom JE, Schober MF, Brennan SE. Disfluency rates in conversation: Effects of age, relationship, topic, role, and gender. Language and speech. 2001;44(2):123-147.

20. Levelt WJM. Monitoring and self-repair in speech. Cognition. 1983;14:41-104.

21. Kapatsinski V. Frequency of use leads to automaticity of production: Evidence from repair in conversation. Language and speech. 2010;53(1):71-105.

22. Nettelbladt U, Hansson K. Mazes in Swedish pre-school children with specific language impairment. Clinical Linguistics and Phonetics. 1999;13:483-497. doi: 10.1080/026992099298997

23. Bedore LM, Fiestas CE, Pena ED, Nagy VJ. Cross-language comparisons of maze use in Spanish and English in functionally monolingual children. Bilingualism: Language and Cognition. 2006;9(3):233-247. doi:dx.doi.org/10.1017/S1366728906002604.

24. Leadholm B, Miller JF. Language sample analysis: The Wisconsin guide. Madison,WI: Department of Public Instruction. 1992.

25. Thordardottir E. Ellis Weismer S. Content mazes and filled pauses on narrative language samples of children with specific language impairment. Brain and Cognition. 2002;48(2-3):587-592

26. Miller JF. The search for the phenotype of disordered language performance. In M. L. Rice (Ed.) Toward a Genetics of Language (Mahwah, NJ: Lawrence Erlbaum) 1996. p. 297-314.

27. Silver MR. A comparison of maze frequency and type across language and speaker: A look at English and Spanish narrative retells. ETD Collection for University of Texas, El Paso. 2015. AAI1591998. http://digitalcommons.utep.edu/dissertations/AAI1591998

28. Gollan TH, Goldrick M. Does bilingualism twist your tongue? Cognition. 2012;125(3):491-497.

29. Sandoval TC, Gollan TH, Ferreira VS, Salmon DP. What causes the bilingual disadvantage in verbal fluency? The dual-task analogy. Bilingualism: Language \& Cognition. 2010;13:231-252.

30. Gleitman LR, Gleitman H, Shipley EF. The emergence of the child as grammarian. In Topics in Cognitive Development 1977. (p. 91117). Springer US.

31. Gollan TH, Montoya RI, Cera C, Sandoval TC. More use almost al- ways means a smaller frequency effect: Aging, bilingualism, and the weaker links hypothesis. Journal of memory and language. 2008;58(3):787-814.

32. Ivanova I, Costa A. Does bilingualism hamper lexical access in speech production? Acta Psychologica. 2008;127(2):277-288. doi: 10.1016/j.actpsy.2007.06.003

33. Rieger CL. Repetitions as self-repair strategies in English and German conversations. Journal of Pragmatics. 2003;35(1):47-69. doi: 10.1016/S0378-2166(01)00060-1.

34. Wiese R. Prosodic conditions on clitics. Phonologica. 1984;331338.

35. Hopper J. Learning two languages: Maze behaviors in narrative discourse for Spanish-English bilinguals. All Graduate Plan B and other Reports. 2014. p. 440. Downloaded from http://digitalcommons.usu.edu/gradreports/440.

36. Evans JL. Self-initated repairs: A reflection of communicative monitoring in young children. Developmental Psychology. 1985; 21(2):365-371.

37. Eckert P. Cooperative competition in adolescent "girl talk". Discourse Processes. 1990;13,91-122. doi:10.1080/01638539009544748.

38. Bialystok E. Metalinguistic aspects of bilingual processing. Annual Review of Applied Linguistics. 2001;21:169-181. doi:10.1016/ S0378-2166(01)00060-1.

39. Bialystok E, Luk G, Peets KF, Yang S. Receptive vocabulary differences in monolingual and bilingual children. Bilingualism: Language and Cognition. 2010;13(4):525-531. doi:10-1017/S1366728909990423.

40. Levelt WJM, Cutler A. Prosodic marking in speech repair. Journal of Semantics. 1983;2(2):205-217.

41. Nooteboom SG. The perceptual reality of some prosodic durations. Journal of Phonetics. 1973;1(1):25-45.

42. Fox-Tree JEF. The effects of false starts and repetitions on the processing of subsequent words in spontaneous speech. Journal of memory and language. 1995;34(6):709-738. doi:10.1006/jmla.1995.1032.

43. Shriberg E, Stolcke A. Word predictability after hesitations: a corpus-based study. In Spoken Language, 1996. ICSLP 96. Proceedings., Fourth International Conference on (1996;3:1868-1871). IEEE. doi:10.1109/1CSLP.1996.607996.

44. Kowal S, Bassett MR, O'Connell DC. The spontaneity of media interviews. Journal of psycholinguistic research. 1985;14(1):1-18. doi:0090-6905/85/0100-0001\$04.50/0.

45. Lickley RJ, Bard EG. On not recognizing disfluencies in dialogue. In Spoken Language, 1996. ICSLP 96. Proceedings., Fourth International Conference on (1996;3:876-1879). IEEE. doi:10.1109/ 1CSLP.1996.607998.

46. Bialystok E, Hakuta K. Confounded age: Linguistic and cognitive factors in age differences for second language acquisition. Second language acquisition and the critical period hypothesis. 1999;161181.

47. Birdsong D, Molis M. On the evidence for maturational constraints in second-language acquisition. Journal of Memory and language. 2001;44(2):235-249. doi:org/10.1006/jmla2000.2750.

48. Flege JE, Yeni-Komshian GH, Liu S. Age constraints on secondlanguage acquisition. Journal of memory and language. 1999;41 (1):78-104. 
49. Han Z, Odlin T. Fossilization in adult second language acquisition. Toronto: Multilingual Matters. 2004.

50. Muñoz C, Singleton D. A critical review of age-related research on L2 ultimate attainment. Language Teaching. 2011;44(1):1-35. doi:org/10.1017/S0261444810000327

51. Johnson JS, Newport EL. Critical period effects in second language learning: The influence of maturational state on the acquisition of English as a second language. Cognitive psychology. 1989;21(1):60-99. doi:org/10.1016/0010-0285(89)90003-0.

52. Long MH. Maturational constraints on language development. Studies in second language acquisition. 1990;12(03):251-285. doi:org/10.1017/S0272263100009165.

53. Pinker S. How could a child use verb syntax to learn verb semantics?. Lingua. 1994;92:377-410. doi:10.1016/0024-3841(94)90347-6.

54. Gollan TH, Acenas LA. What is a TOT? Cognate and translation effects on tip-of the-tongue states in Spanish-English and TagalogEnglish bilinguals. Journal of Experimental Psychology: Learning, Memory and Cognition. 2004;30(1):246-269.

55. Gollan TH, Montoya RI, Bonanni MP. Proper names get stuck on bilingual and monolingual speakers' tip of the tongue equally often. Neuropsychology. 2005;19(3):278-287.

56. Gollan TH, Montoya RI, Werner GA. Semantic and letter fluency in Spanish- English bilinguals. Neuropsychology. 2002;16:562576.

57. Gollan TH, Silverberg NB. Tip-of-the-tongue states in HebrewEnglish bilinguals. Bilingualism: Language and Cognition. 2001;4 (1):63-84.

58. Collier VP. How long? A synthesis of research on academic achievement in a second language, Tesol Quarterly. 1989;23(3):509-531.

59. Hakuta K, Goto Butler Y, Witt D. How long does it take English learners to attain proficiency? Santa Barbara, 2000. CA: University of California Linguistic Minority Research Institute Policy Report.

60. Jacobson PF, Schwartz RG. English past tense use in bilingual children with language impairment. American Journal of SpeechLanguage Pathology. 2005;14(4):313-323. doi:1058-0360/05/14040313.

61. Hammill DD, Brown VL, Larsen SC, Wiederholt JL. Test of adolescent and adult language. Austin, 1994. Texas: Pro-Ed.

62. Goodglass H, Kaplan E, Barresi B. BDAE: The Boston Diagnostic Aphasia Examination. Lippincott Williams and Wilkins. 2001.

63. Pinkney J. Aesop's fables. Chronicle Books. 2000.

64. Boersma P, Weenink D. Praat: Doing phonetics by computer. 2009 (Version 5.1. 05) [Computer program]. Retrieved from https:// praat.secursoft.net/?network=ContentSearch\&utm_source= bing\&utm_medium =cpc\&utm_campaign=US\%3A\%20Audio\& utm_term=Download\%20Praat\&utm_content=praat.

65. Rispoli M. Changes in the nature of sentence production during the period of grammatical development. Journal of Speech, Language, and Hearing Research. 2003;46:818-830.

66. Hilton HE. Annotation and analyses of temporal aspects of spoken fluency. CALICO Journal. 2009;26:644-661.

67. Clark HH, Fox Tree JE. Using uh and um in spontaneous speaking. Cognition. 2002;84(1):73-111. doi:10.1016/S0010-0277(02) 00017-3.
68. McKee C, Rispoli M, McDaniel D, Garrett M. How do children become adult sentence producers?. Applied Psycholinguistics. 2006; 27(1):74-81. doi: org/10.1017/S0142716406060139.

69. Verhoeven LT. Monitoring in children's second language speech. Second Language Research. 1989;5(2):141-155.

70. Fathman AK. Repetition and correction as an indication of speech planning and execution processes among second language learners. Towards a crosslinguistic assessment of speech production. 1980;77-85.

71. Bliss LS, Covington Z, McCabe A. Assessing the narratives of African American children. Contemporary Issues in Communication Science and Disorders. 1999;26:160-167.

72. Justice LM, Bowles RP, Kaderavek JN, Ukrainetz TA, Eisenberg SL, Gillam RB. The index of narra- tive microstructure: A clinical tool for analyzing school-age children's narrative performances. American Journal of Speech- Language Pathology. 2006;15:155-191.

73. Katz L, Champion T. There's no " 1 " way to tell a story. Affirming students' right to their own language: Bridging language policies and pedagogical practices. 2009. (p. 192-205). New York, NY: Routledge.

74. Craig HK, Washington JA. Malik goes to school: Examining the language skills of African American students from preschool-5th grade. Psychology Press. 2006.

75. Ivy LJ, Masterson JJ.A comparison of oral and written English styles in African American students at different stages of writing development. Language, Speech, and Hearing Services in Schools. 2011;42(1):31-40.

76. Mills MT, Watkins RV, Washington JA. Structural and dialectal characteristics of the fictional and personal narratives of schoolage African American children. Language, speech, and hearing services in schools. 2013;44(2):211-223.

77. Ross SH, Oetting JB, Stapleton B. Preterite had+ V-ed: A developmental narrative structure of African American English. American Speech. 2004;79(2):167-193.

78. Schachter RE, Craig HK. Students' production of narrative and AAE features during an emergent literacy task. Language, Speech, and Hearing Services in Schools. 2013;44(3):227-238.

79. Terry NP, Mills MT, Bingham GE, Mansour S, Marencin N. Oral narrative performance of African American prekindergartners who speak nonmainstream American English. Language, Speech, and Hearing Services in Schools. 2013;44(3):291-305.

80. Hughes DL, McGillivray L, Schmidek M. Guide to narrative language: Procedures for assessment. Eau Claire, WI: Thinking Publications. 1997.

81. Bates E. Special issue: Cross-linguistic studies of aphasia. Brain and Language. 1991;41(2).

82. Mandler JM, Johnson NS. Remembrance of things parsed: Story structure and recall. Cognitive Psychology. 1977;9:111-151.

83. Merritt DD, Liles BZ. Narrative analysis: Clinical applications of story generation and story retelling. Journal of Speech and Hearing Disorders. 1989;54:429-438.

84. Schneider P. Effects of pictures versus orally presented stories on story retellings by children with language impairment. American Journal of Speech-Language Pathology. 1996;5:86-96. 
85. Schneider P, Dube RV. Story pre-sentation effects on children's retell content. American Journal of Speech-Language Pa-thology. 2005;14(1):52-60.

86. Stein N, Glenn C. An analysis of story comprehension in elementary school children. In R. O. Freedle (Ed.), New directions in discourse processing. 1979;2:53-120.

87. Allen MS, Kertoy MK, Sherblom JC, Pettit JM. hildren's narrative productions: A comparison of personal event and fictional stories. Applied psycholinguistics. 1994;15(2):149-176.

88. Celinska DK. Narrative Voices of Early Adolescents: Influences of Learning Disability and Cultural Background. International Journal of Special Education. 2009;24(3):150-172.

89. Champion TB. Understanding storytelling among African American children: A journey from Africa to America. Mahwah, NJ: Lawrence Erlbaum Associates. 2003.

90. Labov W. Language in the inner city. Philadelphia: University of Pennsylvania Press. 1972.
91. Peterson C, McCabe A. Developmental psycholinguistics: Three ways of looking at a child's narrative. New York: Plenum Press. 1983.

92. Glenn-Applegate K, Breit-Smith A, Justice LM, Piasta SB. Artfulness in young children's spoken narratives. Early Education and Development. 2010;21(3):468-493.

93. Ukrainetz TA, Gillam RB. The expressive elaboration of imaginative narratives by children with specific language impairment. Journal of Speech, Language, and Hearing Research. 2009;52(4): 883-898.

94. Ukrainetz TA, Justice LM, Kaderavek JN, Eisenberg SL, Gillam RB, Harm HM. The development of expressive elaboration in fictional narratives. Journal of Speech, Language, and Hearing Research. 2005;48(6):1363-1377.

95. Liles BZ, Duffy RJ, Merritt DD, Purcell SL. Measurement of narrative discourse ability in children with language disorders. Journal of Speech, Language, and Hearing Research. 1995;38(2):415-425. 